Documentation et bibliothèques

DOCUMENTATION BIBLIOTHEQQUES

\title{
Henri-Jean Martin, le fondateur de l'École française de l'histoire du livre vient de nous quitter
}

\section{Éric Le Ray}

Volume 53, numéro 2, avril-juin 2007

URI : https://id.erudit.org/iderudit/1029239ar

DOI : https://doi.org/10.7202/1029239ar

Aller au sommaire du numéro

\section{Éditeur(s)}

Association pour l'avancement des sciences et des techniques de la documentation (ASTED)

\section{ISSN}

0315-2340 (imprimé)

2291-8949 (numérique)

Découvrir la revue

\section{Citer ce document}

Ray, É. L. (2007). Henri-Jean Martin, le fondateur de l’École française de l'histoire du livre vient de nous quitter. Documentation et bibliothèques, 53(2), 119-124. https://doi.org/10.7202/1029239ar

Tous droits réservés (C) Association pour l'avancement des sciences et des techniques de la documentation (ASTED), 2007
Ce document est protégé par la loi sur le droit d'auteur. L'utilisation des services d'Érudit (y compris la reproduction) est assujettie à sa politique d'utilisation que vous pouvez consulter en ligne.

https://apropos.erudit.org/fr/usagers/politique-dutilisation/ 


\title{
Henri-Jean Martin, le fondateur de l'École française de l'histoire du livre vient de nous quitter
}

\author{
ÉRIC LE RAY \\ Stagiaire postdoctoral à la Chaire de recherche industrielle Quebecor sur l'impression et les communi- \\ cations graphiques de l'Université du Québec à Trois-Rivières. \\ leraycelte7@sympatico.ca
}

L 'HISTORIEN DU LIVRE FRANÇAIS HENRI-JEAN Martin par qui s'est développée une véritable école française d'histoire du livre, y compris au Québec, par l'intermédiaire de son ami Claude Galarneau, est décédé dans la nuit du 12 au 13 janvier 2007, à l'âge de 83 ans. Il fut l'auteur, en collaboration avec Lucien Febvre, d'un ouvrage remarquable et remarqué, L'apparition du livre, en 1958, qui annonça l'émergence d'une nouvelle filière de recherche universitaire portant sur l'histoire du livre, de l'édition mais aussi de l'imprimé dans son essence la plus générale.

\section{Ses racines familiales}

Henri-Jean Martin est né à Paris le 16 janvier 1924. Sa disparition annonce, comme on le dit en Afrique lorsqu'un vieux sage meurt, celle d'une véritable bibliothèque vivante. En effet, le Français Henri-Jean Martin fut un historien qui, comme le soulignait son ancien élève Claude Jolly ${ }^{1}$, dans un discours prononcé le 13 mai 1997 dans les grands salons du rectorat de Paris à l'occasion de la remise d'un volume de mélanges offert à son intention ${ }^{2}$, occupa une place éminente dans l'espace académique comme conservateur de bibliothèque, professeur et savant. Diplômé de l'École des chartes, Henri-Jean Martin y étudia de 1943 à 1947. Son entrée dans le monde du livre est un concours de circonstances car, au départ, il se destinait à une carrière scientifique et son milieu familial témoignait traditionnellement d'une certaine hostilité à l'égard des intellectuels. Il fut élève au cours Hattemer, où les enfants étaient suivis par une institutrice personnelle. Il n'allait en classe qu'une fois par semaine. C'est dans la bibliothèque familiale qu'il va découvrir et apprendre à aimer la lecture en compagnie de sa mère qui se charge de son instruction. Sa culture lui vient donc davantage de ses lectures que de l'école. Il découvrira l'histoire par le jeu, en reconsti-

1. Claude Jolly, "Henri-Jean Martin, bibliothécaire, professeur et savant ", (texte du discours), Paris, Bulletin des bibliothèques de France, t. 42, $\mathrm{n}^{\circ} 6,1997$, p. 82-84. Claude Jolly est sous-directeur chargé des bibliothèques au ministère de l'Éducation nationale.

2. Henri-Jean Martin: Le livre et l'historien. Études offertes en l'honneur du professeur Henri-Jean Martin, réunies par Frédéric Barbier, Annie Parent-Charon, François Dupuigrenet Desroussilles, Claude Jolly, Dominique Varry, Genève, Droz, 1997. tuant des batailles historiques avec des soldats de plomb qu'il aime fabriquer lui-même.

Le grand-père maternel d'Henri-Jean Martin était orfèvre et son père, issu de la deuxième promotion de l'École supérieure d'électricité, était un inventeur. Ce dernier expérimenta les premières locomotives électriques et entra dans le monde du cinéma en tant que premier ingénieur engagé par Léon Gaumont, l'un des pères du cinéma français. Il va ainsi transmettre à son fils le goût et la passion pour les communications et la recherche, avec les difficultés financières que cela implique parfois, lorsque les découvertes ou le fruit des recherches n'ont pas le succès escompté. Henri-Jean Martin est donc tenté un moment par l'école d'ingénieur ou la carrière d'officier.

La défaite de la France pendant la Seconde Guerre mondiale et le fait que son père tombe gravement malade vont décider Henri-Jean Martin, alors au lycée Henri-IV, y préparant l'École normale supérieure en hypokhâgne, à orienter sa carrière vers l'École des chartes. Le concours se préparait précisément à HenriIV, dans une classe jumelée à l'hypokhâgne. Il va garder de cette période un goût prononcé pour le bricolage et la réalisation de maquettes et de soldats de plomb, ce qui va lui permettre tout naturellement d'apparaître plus tard comme le spécialiste des techniques du livre. Il en gardera aussi un intérêt marqué pour la recherche universitaire, qu'il mit un moment entre parenthèse pour s'occuper de sa famille en difficulté, avant d'écrire sa thèse en 1969.

\section{Des rencontres}

De nombreuses rencontres vont l'influencer par la suite, notamment avec le professeur de philosophie Alquier, en hypokhâgne; ou encore, à l'École des chartes, le professeur d'histoire moderne et contemporaine Métivier et le conservateur Pierre Marot, qui enseignait l'archivistique, la bibliographie et l'histoire du livre. Ce dernier va lui faire découvrir le monde des imprimeurs célèbres. Henri-Jean Martin suivra aussi certains cours à l'École pratique des hautes études, dont ceux d'Émile Coornaert, professeur au Collège de France. Cet économiste, avec un conservateur des Archives notariales de 


\section{L'ignorance en matière d'histoire du livre était totale, de même que la connaissance des précautions critiques à prendre pour pouvoir éditer sérieusement un texte à partir d'un livre imprimé ancien.}

$\alpha<\alpha<\alpha<\alpha<\alpha<\alpha<\alpha<\alpha<\alpha<\alpha<\alpha<\alpha<\alpha<\alpha<\alpha<\alpha<\alpha<\alpha<\alpha<\infty<\infty$

Paris à la retraite, Ernest Coyecque, va lui faire découvrir le monde des corporations par une approche économique de l'histoire de France. Ils feront de lui un historien du livre, puisque c'est à leur contact qu'Henri-Jean Martin décidera d'étudier l'économie de l'édition puis, par la suite, de préparer une thèse de doctorat d'État. Henri-Jean Martin rencontre enfin Julien Cain, administrateur général à la Bibliothèque nationale, qui sera membre de son jury de thèse à l'École des chartes, thèse qu'il soutient avec succès, en 1947 , pour d'obtention d'un diplôme d'archiviste-paléographe.

Julien Cain fera entrer Henri-Jean Martin à la Bibliothèque nationale car son travail sur Eustache Le Noble, polémiste de l'époque de Louis XIV, le conduit à une étude très matérielle de la production imprimée de ce personnage. Il se spécialise dans l'identification des adresses d'éditions falsifiées et clandestines, ce qui lui donne une réputation de spécialiste des techniques du livre, ce qui semble plaire à Julien Cain. Henri-Jean Martin sera alors affecté à la Réserve des imprimés, où l'on conserve les livres rares et précieux publiés depuis l'invention de la typographie à caractères mobiles.

\section{L'histoire du livre}

L'histoire du livre, à l'époque où Henri-Jean Martin commence à s'intéresser à ce sujet, est alimentée par des érudits locaux, des imprimeurs qui s'intéressent à l'histoire de leur profession et, enfin, des bibliophiles et des passionnés de reliure. Du côté des universitaires, comme il le dit lui-même dans ses entretiens accordés à Jean-Marc Chatelain et Christian Jacob, à l'automne 2002, et sur lesquels nous appuyons notre article, "l'ignorance en matière d'histoire du livre était totale, de même que la connaissance des précautions critiques à prendre pour pouvoir éditer sérieusement un texte à partir d'un livre imprimé ancien ${ }^{3} »$. Ces techniques étaient déjà bien connues dans le monde anglo-saxon et portaient le nom de "bibliographie matérielle", des techniques nées de l'étude des œuvres de Shakespeare au lendemain de la Première Guerre mondiale. D’après Henri-Jean Martin, le mouvement a pénétré en France après la Seconde Guerre mondiale à la fois par la voie de bibliothécaires australiens, comme Wallace Kirsop, de professeurs français comme Roger Laufer, et par l'inter-

3. Henri-Jean Martin, Les métamorphoses du livre, entretiens avec Jean-Marc Chatelain et Christian Jacob, Paris, Albin Michel, coll. «Itinéraires du savoir, » 2004, 297 pages. médiaire de chercheurs d'Oxford comme Giles Barber. Cette démarche fut reprise par Jeanne Veyrin-Forrer qui fut "pendant des décennies, [...] la prêtresse française de la bibliographie matérielle ${ }^{4}$. Pour Henri-Jean Martin, cette ignorance s'explique par le fait que l'université française s'est reconstituée pratiquement sans bibliothèque après la rupture de l'époque révolutionnaire. L'université française se serait donc développée d'une façon purement rhétorique, sans ancrage dans le monde du livre et de l'imprimé. Au cours de son séjour à la Bibliothèque nationale, Henri-Jean Martin, en plus de son travail de classification, va organiser plusieurs expositions, dont une portant sur l'histoire de l'Imprimerie royale - puis nationale - , qui va lui permettre de retrouver et d'assurer la sauvegarde des cuivres et des bois gravés qui avaient été utilisés pour l'illustration et l'ornementation des livres de cet établissement du $\mathrm{xVII}^{\mathrm{e}}$ au $\mathrm{XIX}^{\mathrm{e}}$ siècle. "Ce matériel inestimable se trouvait alors emballé dans des paquets rangés au-dessus des vestiaires des ouvriers ${ }^{5}$." Cette exposition, ainsi que son intervention pour sauver les papiers de Philippe Renouard, descendant d'un autre érudit, Antoine-Augustin Renouard, et pour sauver le catalogage des ouvrages concernant les auteurs, les anonymes ou les incunables semble avoir permis de donner une impulsion capitale à l'histoire du livre en France.

Agacé par les lenteurs de l'administration de la Bibliothèque nationale, Henri-Jean Martin est affecté un moment au catalogage de l'Enfer (livres érotiques ou pornographiques) avant de se révolter contre sa situation et d'être encouragé à demander un détachement au CNRS afin de poursuivre sa thèse. Il hésite un moment à partir en Amérique du Nord rejoindre son ami Claude Galarneau, au Québec, puis il obtient un poste de directeur à la bibliothèque municipale de Lyon, en 1962, la plus importante bibliothèque de province à l'époque.

\section{L'apparition du livre ou l'apparition de l'imprimerie ?}

C'est Émile Coornaert et Fernand Braudel qui vont permettre le rapprochement entre Henri-Jean Martin et Lucien Febvre (1878-1956). "Émile Coornaert m'avait signalé un jour que quelqu'un commençait à travailler sur la corporation des libraires et des imprimeurs parisiens, sujet auquel je voulais consacrer ma thèse de doctorat. J'ai donc décidé de faire rapidement une thèse des Hautes Études pour retenir le sujet ${ }^{6}$. " Lucien Febvre, historien consacré, père fondateur des Annales, était à la retraite et réfléchissait à un ouvrage consacré au livre à la Renaissance, dont l'imprimerie à caractères mobiles devait être le thème central, ainsi que le processus économique et culturel qui permit l'émergence du livre imprimé. Il demanda à Henri-Jean Martin de se 
joindre à sa recherche, qui devait aussi intégrer une réflexion sur les conséquences "psychologiques et intellectuelles " d'une telle apparition. Lucien Febvre, après avoir fourni un plan à Henri-Jean Martin, avec ces deux célèbres formules choc "le livre, cette marchandise " et "le livre, ce ferment ", meurt quelque temps plus tard, le 27 septembre 1956, sans que l'ouvrage prévu soit remis à l'éditeur. $\mathrm{M}^{\mathrm{me}}$ Febvre et Fernand Braudel vont insister pour que l'ouvrage soit publié rapidement avec les deux noms et préfacé par Braudel lui-même. Henri-Jean Martin avait 30 ans lorsqu'il a commencé à écrire L'apparition du livre - il y travaillera jusqu'en 1957 -, un titre trompeur, rappelle son auteur, car il s'agit en fait de l'apparition de l'imprimerie. Cet ouvrage va cependant marquer l'historiographie française même si, au moment de sa parution en 1958, il n'eut aucun retentissement immédiat. Aujourd'hui, il est devenu un classique, souvent réédité, et traduit en plusieurs langues.

Henri-Jean Martin va y développer, ce qui va rester une constante dans ses recherches par la suite, un intérêt pour l'évolution des faits de civilisation à travers l'évolution des habitudes mentales et les modifications de l'outillage mental comme conséquences de l'apparition du livre imprimé et de l'imprimerie, avant de s'intéresser à l'histoire de l'écriture et de la lecture. Il va y présenter, par exemple, les mécanismes de fabrication et de diffusion du manuscrit et la naissance d'une industrie papetière à la veille de l'apparition de l'imprimerie, qui auront des conséquences sur ces mécanismes et permettront cette apparition comme "conditions préalables ». Des conséquences qui vont se répercuter à long terme, jusqu'à nos jours, notamment en mettant en perspective les problèmes techniques qui conditionnent le travail intellectuel ainsi que le lien entre les progrès de la circulation matérielle des informations et les progrès de la communication des idées.

À travers l'histoire des imprimeurs, Henri-Jean Martin va pouvoir retracer l'itinéraire de la pénétration des idées nouvelles, montrer l'impact qu'a eu sur les esprits l'accessibilité à tous les textes sacrés et montrer, enfin, comment l'imprimerie a favorisé la fixation du vocabulaire et de l'orthographe des langues nationales, l'essor de littératures modernes dans ces langues, mais aussi de littératures scientifiques et techniques. Les conséquences de la mutation de la communication au $\mathrm{XV}^{e}$ siècle, explique Elizabeth Eisenstein 7 , ont été le passage du manuscrit au livre imprimé, que l'auteure associe à la transition du Moyen Âge au début des temps modernes, au cours desquels s'est développée la recherche scientifique et technique pour aboutir aux différentes révolutions industrielles modernes et contemporaines. "Lorsqu'ils tentent d'expliquer les nouvelles interactions entre la théorie et la pratique, entre l'homme d'école et l'artisan, bien peu de spécialistes mentionnent l'avènement de l'imprimerie. Ce fut pourtant cette invention

7. Elizabeth L. Eisenstein, La révolution de l'imprimé dans l'Europe des premiers temps modernes, Paris, Éditions la découverte, 1991, 355 pages.

\section{A travers l'histoire des imprimeurs, Henri-Jean Martin va pouvoir retracer l'itinéraire de la pénétration des idées nouvelles.}

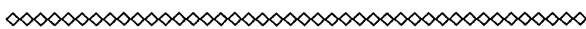

qui rendit les livres plus accessibles aux artisans, et les manuels pratiques plus accessibles aux érudits. Ce fut cette invention qui encouragea artistes et ingénieurs à publier des traités théoriques, et poussa les professeurs à traduire des ouvrages techniques. ${ }^{8}$. "L'âge de l'imprimé est aussi paradoxalement le temps d'une affirmation du principe d'autorité plutôt que celui d'une libération, le temps d'une sacralisation de la figure de l'auteur et surtout celui d'un renforcement de la loi écrite, fixée et même figée par l'écrit, aux dépens de la tradition le plus souvent orale et donc en évolution constante. On assiste aussi à des phénomènes de cristallisation et de normalisation autour d'une langue nationale au détriment de dialectes, ou autour d'oppositions à une religion ou à un régime politique, grâce aux réseaux d'imprimeries clandestines. L'âge de l'imprimé apparaît ainsi comme un "univers à finalité ouverte".

Henri-Jean Martin reconnaît cependant que la plus grande lacune de cet ouvrage en collaboration avec Lucien Febvre est de ne pas avoir parlé des auteurs et des pratiques de lecture ni expliqué dans quel contexte économique et social l'art typographique est né en Allemagne et dans quelle mesure cette naissance correspondait à une nouvelle demande de textes, un renouveau économique, et était liée aux progrès techniques. HenriJean Martin y répondra plus tard dans un autre livre important, Histoire et pouvoirs de l'écrit, ou dans celui sur La naissance du livre moderne, en étudiant l'organisation des textes. D'autres chercheurs,

Martin Lowry et Jean-François Gilmont, notảmment, poursuivront ses recherches sur «l'outillage mental » des sociétés et les rapports que la parole et l'écriture - ces deux modes de communication entretiennent entre elles au sein de ces sociétés, ainsi que leurs répercussions sur l'évolution de la pensée et du support de cette pensée. Les travaux de Roger Chartier vont s'orienter sur le sens du texte en rapport avec sa matérialité, sa mise en forme et à sa mise en livre (Histoire de la lecture dans le monde occidental), car la transmission d'un texte suppose sa dématérialisation et sa re-matérialisation incessantes, soit techniquement et matériellement, soit mentalement et culturellement. Son ancien élève et ami, Frédéric Barbier, dans son fameux ouvrage L'empire du livre, analysera, par exemple, la montée du nationalisme allemand empruntant les

8. Elizabeth L. Eisenstein, «Le livre et la culture savante », dans Histoire de l'édition française, tome I, Paris, Fayard, 1989, p. 674. 


\section{Sa thèse consiste à étudier l'évolution de la production imprimée sur le plan économique comme sur le plan intellectuel.}

$\infty<0<0<0<0<0<0000000000000000000000000000$

réseaux de librairies. Il élargit sa pensée dans son dernier livre L'Europe de Gutenberg, le livre et l'invention de la modernité occidentale.

\section{Passage à Lyon}

À son arrivée à Lyon en 1962, alors qu'il poursuit la rédaction de sa thèse, Henri-Jean Martin se lance dans un vaste projet de construction d'une nouvelle bibliothèque et veille à accroître les ressources en personnel de cette bibliothèque, qu'il qualifie "d'assoupie", qui passeront de 20 à 120 employés. Son double statut de responsable d'un service municipal - la bibliothèque appartient à la Ville de Lyon - et de fonctionnaire de l'État lui permet de faire avancer ses dossiers rapidement et d'obtenir des subventions substantielles pour aller au bout de ses projets. Il instaure aussi une politique de lecture publique dynamique, en faisant installer une bibliothèque par quartier dans Lyon, et soutient la réalisation de grandes enquêtes sociologiques sur les pratiques de lectures afin de relancer la lecture en France - car les Français ne lisent pas. Il s'occupe également de l'informatisation des bibliothèques.

L'existence de la nouvelle bibliothèque va contribuer à enrichir les sources d'informations et d'études des universités locales. Elle va aussi attirer dans la ville non seulement des grandes écoles comme l'École normale supérieure, dite de Saint-Cloud (sections lettres et sciences humaines), et l'École nationale supérieure des sciences de l'information et des bibliothèques (ENSSIB), mais aussi des chercheurs de haut niveau. Henri-Jean Martin va se faire un point d'honneur d'attirer des chercheurs de qualité et de faciliter leur travail en améliorant leur environnement de recherche et leur accueil afin de les retenir à Lyon. Il va aussi développer une politique d'exposition et contribuer à la création du Musée de l'imprimerie de Lyon, en association avec la bibliothèque, avec l'aide du libraire parisien André Jammes et du maître imprimeur lyonnais Maurice Audin. Le musée est conçu sur le modèle du Musée Gutenberg, comme un centre de recherche et un espace muséographique, explique Henri-Jean Martin qui ajoute : «Ce musée devait à la fois présenter un patrimoine typographique et répondre à un objectif didactique : expliquer comment un livre était fabriqué et quelles étaient les différentes techniques mises en œuvre ${ }^{9}$."

9. Henri-Jean Martin, op. cit., p. 112.
Ce musée tente aujourd'hui de développer de multiples collaborations avec l'ENSSIB, l'École normale supérieure, l'École des chartes, les bibliothèques municipales de Lyon et les universités lyonnaises. Il est devenu musée national en 2005 et est dirigé par l'historien d'origine écossaise Alan marshall, spécialiste de l'histoire de la photocomposition. Ce dernier encourage la collecte de fonds historiques et d'archives professionnelles comme celles d'Higounet et de Moyroud, les inventeurs français de la photocomposition, ou celles de la société fondée par Hippolyte-Auguste Marinoni (1823-1904) vers $1850^{10}$. Ce dernier est le père des presses rotatives françaises avec clichés stéréotypiques, mises au point à partir des recherches du Français d'origine allemande Jacob Worms, dès 1845, à l'initiative d'Émile de Girardin et considérées par les professionnels et les historiens comme le principe industriel qui va permettre l'émergence des médias de masse au XIX ${ }^{\mathrm{e}}$ siècle avec, en particulier, une presse populaire unique au monde autour du Petit Journal et du Petit Parisien avec des tirages dépassant le million d'exemplaires.

\section{Le professeur et le savant}

Henri-Jean Martin touche à l'enseignement dès 1954 en créant une préparation au concours de l'École des chartes à l'École alsacienne où il enseigne, mais cette nouvelle préparation n'aura pas de suite. En 1958, il enseigne l'histoire du livre dans le programme technique des bibliothécaires, puis en 1962 à l'École nationale supérieure de bibliothécaires (ENSB), devenue aujourd'hui l'ENSSIB. Pendant son séjour à Lyon, en 1963, Henri-Jean Martin est élu directeur d'études de la IV ection de l'École Pratique des Hautes Études (EPHE). Il y restera plus de 30 ans. Chaque semaine, pendant huit ans, il quittait Lyon le dimanche soir pour aller donner à Paris, le lundi, quatre heures de cours à l'École des bibliothèques et à l'École Pratique des Hautes Études, et rentrer le soir à minuit à Lyon. Il y enseigne d'une part la bibliographie, d'autre part les techniques et l'histoire du livre et de la gravure. À partir de 1968, on propose à Henri-Jean Martin de prendre la direction d'une chaire de bibliographie et d'histoire du livre à l'École nationale des chartes, une chaire spécialement créée pour lui. Il retourne donc à Paris où il va pouvoir finir sa thèse qu'il soutiendra en 1968, et publiera en 1969 sous le titre Livre, pouvoirs et société à Paris au XVIII siècle (1598-1701), dix ans après avoir publié un grand ouvrage de synthèse aux côtés d'un des grands maîtres des études historiques de l'université française. Sa thèse consiste à étudier l'évolution de la production imprimée sur le plan économique comme sur le plan intellectuel. La première partie est consacrée aux données statisti-

10. Ces derniers fonds ont été réunis à l'initiative d'Éric Le Ray lorsqu'il entreprit d'écrire sa thèse sur ce personnage avec Frédéric Barbier qui dirige aujourd'hui la $\mathrm{IV}^{\mathrm{e}}$ section de l'École pratique des hautes études (EPHE), succédant à Henri-Jean Martin. 
ques et à l'évolution de la production, les deux autres à l'étude du métier, à l'analyse des bibliothèques privées et à l'interprétation globale du mouvement du siècle, de l'époque baroque à l'époque classique, en fonction de la conjoncture économique et sociale. Henri-Jean Martin recevra une médaille d'argent du CNRS à la suite de la publication de sa thèse. L'histoire du livre dans son séminaire se développera en direction de l'histoire économique du livre ainsi que vers l'histoire intellectuelle.

\section{L'École française de l'histoire du livre}

Son séminaire va devenir l'un des lieux où va se créer une École française de l'histoire du livre, dont les représentants ont constitué, dans les années 1980, la majorité de la quelque centaine de collaborateurs ayant travaillé à la grande entreprise de L'histoire de l'édition française $e^{11}$ avec Roger Chartier. C'est à cette époque en effet qu'il commence à travailler avec Roger Chartier qui suit son séminaire à la IV ection de l'EPHE tout en appartenant à la $\mathrm{VI}^{\mathrm{e}}$ section (issue de la $\mathrm{IV}^{\mathrm{e}}$ et qui deviendra l'EHESS). Henri-Jean Martin a connu Roger Chartier en 1966 lorsque ce dernier était étudiant à Lyon, sa ville d'origine, et le retrouvera assistant à la Sorbonne. Celui-ci va lancer tout un mouvement de recherche sur la lecture, et compléter ainsi les recherches d'Henri-Jean Martin. La collaboration très active avec Roger Chartier, qui devient successivement maitre-assistant puis directeur de recherches à l'École des hautes études en sciences sociales ( $\mathrm{VI}^{\mathrm{e}}$ section), va encourager Henri-Jean Martin à sortir des considérations avant tout bibliographiques ou techniques en orientant ses recherches vers l'histoire de l'écriture et de la lecture. Roger Chartier s'intéresse, en effet, à la réception individuelle des ouvrages et aux structures mentales, des recherches qui vont susciter un véritable renouvellement de la manière de voir l'histoire littéraire ainsi que l'histoire culturelle et ses pratiques. Roger Chartier lui fera rencontrer aussi Daniel Roche, un spécialiste des Lumières et de la communication sociale, qui est au Collège de France comme lui aujourd'hui, et les Américains Robert Darnton et Elizabeth Eisenstein. Une collaboration internationale s'amorce ainsi doucement, marquée par la venue de professeurs américains, australiens, anglais ou allemands en sabbatique. En 1993, Henri-Jean Martin va prononcer une série de conférences aux États-Unis dans le cadre des Schouler

11. En voici quelques-uns : Jeanne Veyrin-Forrer, chargée de conférence, viendra avec ses élèves, à la fois bibliotécaires et ingénieur du CNRS, Albert Labarre, Brigitte Moreau, Ursula Baurmeister, Bruno Neveu, Raymond Birn, le père dominicain Michel Saffray, les élèves de l'École supérieure de bibliothécaires avant son transfert à Lyon, des normaliens, des professionnels des industries graphiques comme Alphonse Dain, des chartistes comme Denis Pallier, Annie Charon, Anne Sauvy, Motoko Sékiné (aujourd'hui Ninomiya), Micheline Lecoq, Ezio Ornato, Dominique Coq, Jean-Domnique Mellot, Valérie Tesnière, Jean-Marc Chatelain Marianne Grivel, Isabelles Jammes, Pierre Petitmengin, Jacques Breton, Dominique Varry, Frédéric Barbier, qui lui succédera comme directeur d'études et qu'il orienta vers l'histoire de l'édition au $\mathrm{XIX}^{\mathrm{e}}$ siècle en lui faisant faire sa thèse sur la maison Berger-Levrault. Ce dernier fera aussi une thèse de III $^{\mathrm{e}}$ cycle sur l'impri merie strasbourgeoise de la fin du XviIr ${ }^{\mathrm{e}}$ siècle à 1870 avec Daniel Roche et son doctorat d'État sur l'essor de l'imprimerie dans l'empire Wilhelmien entre 1870 et 1914 avec l'économiste François Caron.

\section{L'histoire du livre dans son séminaire se développera en direction de l'histoire économique du livre ainsi que vers l'histoire intellectuelle.

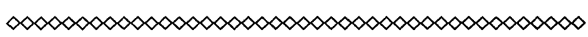

Lectures à l'Université John Hopkins à Baltimore, qui ont été publiées sous le titre The French Book : Religion, Absolutism, and Readership, 1585-1715, ouvrage préfacé par l'historien américain Orest Ranum. Ainsi s'organise l'histoire du livre "à la française " qu'HenriJean Martin fait évoluer vers la période industrielle, en abordant le XIX ${ }^{e}$ siècle. Il crée, en lien avec sa chaire des Hautes Études, la collection « Histoire et civilisation du livre ", aux Éditions Droz, ce qui va lui permettre de faire publier beaucoup de thèses ou de travaux menés sous sa direction. Aujourd'hui, à l'initiative de Frédéric Barbier, une revue du même nom vient de paraître en remplacement de l'ancienne Revue française d'histoire $d u$ livre et une nouvelle perspective est offerte avec la création d'un Institut d'histoire du livre auquel participent ensemble l'ENSSIB, l'École des chartes, le Musée de l'imprimerie de Lyon, l'Université de Lyon-II et l'École pratique des hautes études.

\section{L'histoire de l'édition française}

De 1983 à 1986, Henri-Jean Martin fait paraître en quatre volumes successifs, aux Éditions du Cercle de la librairie, L'histoire de l'édition française, avec Roger Chartier. Ils vont y intégrer dans un même propos un ensemble de points de vue différents sur le livre en matière d'histoire technique et matérielle de la production du livre, d'histoire sociale, économique et institutionnelle de sa diffusion, et enfin d'histoire symbolique et culturelle de sa réception. Cette entreprise put se réaliser à l'initiative d'un éditeur, ancien journaliste au Monde, du nom de Jean-Pierre Vivet. Pascal Fouchet, qui prendra sa succession au Cercle de la librairie, dirigera la réalisation du cinquième volume qui portera sur la deuxième partie du $\mathrm{xx}^{\mathrm{e}}$ siècle. Cette Histoire de l'édition française a provoqué la naissance, un peu partout, de projets d'histoires nationales du livre comme en Angleterre, en Espagne, au Portugal, en Europe Centrale et récemment au Canada avec L'histoire du livre et de l'imprimé au Canada en trois volumes ${ }^{12}$.'Toutes ces démarches semblent avoir un point en commun : conserver ou retrouver, à l'ère du média électronique et d'Internet, une conscience commune identitaire culturelle ou géographique en crise à travers l'histoire du livre. Mais comme le souligne Henri-Jean Martin dans ses

12. Autour de l'équipe éditoriale suivante : Patricia Fleming, Yvan Lamonde, Gilles Gallichan, Fiona Black, Carole Gerson et Jacques Michon. 


\section{Pour Henri-Jean Martin, le livre imprimé s'est d'abord efforcé d'apparaître comme le fac-similé du manuscrit, avant de trouver très lentement une mise en texte qui lui était mieux appropriée.}

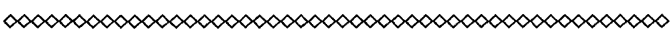

entretiens, "il faudrait replacerl'histoire du livre période par période, et cela depuis la Haute Antiquité, dans le cadre du système global de communication de la société correspondante $^{13}$ ", en faisant appel aux spécialistes des diverses sciences humaines et de la neurophysiologie. C'est un peu ce qu'il fera en orientant ses recherches sur l'histoire de l'écriture et de la lecture, et en publiant son livre Histoire et pouvoirs de l'écrit en 1988. L'idée de réfléchir à l'histoire de l'écriture, née de l'image, et au rôle que celle-ci a pu jouer dans l'évolution des formes de pensée s'est développée chez Henri-Jean Martin avec le temps. En particulier en essayant de reconstituer les attitudes mentales en fonction de l'organisation du texte et en montrant les pouvoirs que l'écrit et l'exercice de l'autorité par l'écriture conféraient à certaines catégories sociales et les liens entre cette écriture et la parole. "Cela devrait être fait non seulement dans le cadre d'une œuvre et d'un auteur, mais dans celui d'une étude générale sur les systèmes de communication d'une société en une époque donnée ${ }^{14}$. " Henri-Jean Martin va recevoir le grand prix Gobert de l'Académie française sur l'intervention de Georges Duby pour cet ouvrage qui sera complété par celui sur Mise en page et mise en texte du livre manuscrit, en 1990, et sur La naissance du livre moderne, publié en 2000, dans lequel il va étudier l'articulation entre la connaissance des textes et la conscience de la matérialité dans laquelle elles se présentent aux lecteurs. Dans ce dernier ouvrage, Henri-Jean Martin va y développer à travers l'étude du geste, des codes graphiques et de la structuration visuelle, une histoire de la mise en texte, de l'organisation des textes, plutôt qu'une histoire de la lecture à travers ses pratiques et ses effets sociaux, laissant ces aspects de la recherche à Roger Chartier ou à Daniel Roche. C'est ainsi, rejoignant en cela les anciennes conclusions de marshall McLuhan avec son "medium is message", en confondant peut-être le sens avec l'usage, qu'il apprendra que la généralisation de la lecture silencieuse n'est pas le fait de l'apparition de l'imprimerie, mais bien le fait du début de la séparation des mots en Europe à partir du $\mathrm{x}^{\mathrm{e}}$ et du $\mathrm{XI}^{\mathrm{e}}$ siècles.

\section{Son dernier livre}

Dans son dernier livre, qu'il a su achever malgré la maladie et la souffrance, avant sa mort, cela ne surprendra personne qu'il soit remonté plus haut dans le temps pour nous raconter une histoire de très longue durée sur la communication humaine. Elle commence avec homo sapiens, au moment où domine la pensée orale, et s'arrête avec l'invention de l'écriture alphabétique, au moment où apparaitt la pensée visuelle. C'est un livre qu'il avait en chantier depuis plusieurs années et qui correspond bien aussi à l'évolution de ses centres d'intérêts passant de l'analyse du support imprimé et des modes de communication et de pensée associés avec leurs productions à une époque et à un lieu donnés, à l'analyse du contenu, l'écriture en remontant vers la lecture puis la parole. Chaque mode et support de communication s'inspire chacun l'un de l'autre avant de prendre une certaine autonomie et d'évoluer à nouveau vers autre chose. Pour Henri-Jean Martin, le livre imprimé s'est d'abord efforcé d'apparaitre comme le fac-similé du manuscrit, avant de trouver très lentement une mise en texte qui lui était mieux appropriée. Assisterons-nous au même phénomène avec l'écran et l'électronique ? Faudra-t-il préserver la mémoire des textes avec leurs matérialisations antérieures ? L'ère numérique nous oblige-t-elle à promouvoir une nouvelle forme d'histoire des textes, une nouvelle forme d'histoire du livre et des bibliothèques ? Quelles seront les répercussions de l'usage de l'ordinateur et d'Internet sur l'avenir du livre et sur les systèmes de pensée et sur la mémoire humaine ? Henri-Jean Martin nous laisse avec de nombreuses pistes de recherche qu'il a ouvertes avec intelligence et humilité. À chacun d'entre nous de suivre le chemin qu'il a ouvert afin de mieux comprendre l'évolution de la structuration mentale de l'être humain dans son histoire et son cheminement vers la conscience et la liberté, une structuration qui a un impact sur la manière de lire et de communiquer et sur la "mise en texte " ou la mise en "lisibilité " d'une pensée. Poursuivre ses réflexions sera la meilleure façon de lui rendre hommage en gardant de lui cette image d'un « tempérament anticonformiste » et généreux. ( $)$ 